\title{
Kommentar zur thüringischen Verfassung: bleibende Erinnerung an Joachim Linck
}

\author{
Linck, Joachim, Manfred Baldus, Joachim Lindner, Holger Poppenhäger, Matthias Ruffert \\ (Hrsg.): Die Verfassung des Freistaats Thüringen. Handkommentar, Nomos Verlagsgesellschaft, \\ Baden-Baden 2013, 1.252 Seiten, € 98,-.
}

Pünktlich zu seinem 20. Jubiläum erschien die Neuauflage des Kommentars der Verfassung des Freistaats Thüringen. Die erste Auflage aus der Zeit kurz nach dem In-Kraft-Treten der Verfassung verstand sich noch als ein Wegweiser durch verfassungsrechtliches Neuland eines Staates, dessen Institutionen sich im Aufbau befanden. ${ }^{1}$ Diese Phase ist lange abgeschlossen. Für die Neuauflage hat Joachim Linck erneut namhafte Herausgeber um sich geschart: den Thüringer Justizminister Holger Poppenhäger, den Präsidenten des Verfassungsgerichtshofs Joachim Lindner sowie die Professoren Manfred Baldus und Matthias Ruffert. Gemeinsam mit ausgewiesenen Autoren aus Wissenschaft und Praxis legen sie einen Kommentar vor, der die mittlerweile zahlreichen verfassungsgerichtlichen Entscheidungen und wissenschaftlichen Veröffentlichungen einarbeitet, die die Auslegung und Anwendung der Verfassung Thüringens betreffen. Joachim Linck ist kurz vor der Fertigstellung des Kommentars unerwartet verstorben. Als Direktor des Thüringer Landtags, Hochschullehrer und Autor, der auch dem Vorstand der Deutschen Vereinigung für Parlamentsfragen angehörte, verband er Wissenschaft und Praxis des Parlamentarismus wie nur wenige andere miteinander. Seiner Frau Inge Linck gebührt das Verdienst, durch die Übernahme der redaktionellen Schlussarbeiten das rechtzeitige Erscheinen des Kommentars sichergestellt zu haben.

Die Kommentierungen sind durchgehend nach einem einheitlichen Schema gegliedert. Das erleichtert die Lesbarkeit und Orientierung erheblich. Die Ausführlichkeit der Kommentierung entspricht der jeweiligen Bedeutung der einzelnen Artikel in der Praxis.

Linck hat einen Großteil der parlamentsrechtlichen Artikel kommentiert. Die plebiszitären Elemente der Thüringer Verfassung bezeichnet er als Bereicherung und Optimierung des parlamentarischen Regierungssystems, weil sie dem Verlust an Volksnähe der Repräsentanten entgegenwirkten. Dem entspricht, dass er demokratische Repräsentation nicht bloß als formal-technische Vermittlung demokratischer Legitimation versteht, sondern inhaltlich ergänzt durch die Anforderung an die demokratischen Repräsentanten, treuhänderisch und uneigennützig für und im Interesse des Volkes gemeinnützig zu handeln. Das Ziel demokratische Repräsentation sei es, ein Höchstmaß an freiwilliger Akzeptanz der Bürger bei einem Minimum an staatlicher Gewalt zu erreichen. Dazu müssten die Bürger darauf vertrauen können, dass ihre Repräsentanten ihre Amtstätigkeit im Interesse des Gemeinwohls ausüben. Deshalb hält Linck Umfrageergebnisse, wonach die Deutschen keiner anderen Berufsgruppe so wenig trauen wie Politikern, für in hohem Maße besorgniserregend im Hinblick auf die Funktionstüchtigkeit der repräsentativen Demokratie. Zum Aufbau von Vertrauen empfiehlt er unter anderem den Ausbau der direkten Demokratie, die Einführung von Teilzeitabgeordneten und die Abschaffung der Immunität der Parlamentarier. Man kann Linck entgegenhalten, dass der demokratische Rechtsstaat auf institutionalisiertem Misstrauen gegenüber den Inhabern politischer Macht beruht. Ausdruck dafür sind insbesondere die zahlreichen „checks and balances“, mit denen sich die staatlichen Gewal- 
ten gegenseitig hemmen und kontrollieren, die Grundrechte, die auch die demokratisch gewählte Mehrheit daran hindern, ihren Willen unbegrenzt durchzusetzen, oder die politischen Grundrechte wie Meinungs-, Presse- und Versammlungsfreiheit. Gesunde Skepsis ist eine Voraussetzung für politische Urteilsfähigkeit und Partizipation. Linck ist allerdings zuzustimmen, dass die Demokratie Vertrauen braucht, und zwar in erster Linie in die politischen Institutionen, also in das System demokratischer Herrschaft, weniger in die handelnden Personen selbst. Aber da die demokratischen Institutionen nicht von den in ihnen handelnden Personen zu trennen sind, ist ein Mindestmaß an Vertrauen auch in die Repräsentanten der Demokratie unumgänglich. Dazu gehört, wie Linck zu Recht bemerkt, neben der gemeinwohlorientierten Amtsführung die Kommunikation und Rückkopplung zwischen dem Volk und seinen Repräsentanten.

Linck konstatiert einen fortschreitenden Machtverlust der Landesparlamente, der durch die beiden Föderalismuskommissionen nicht aufgehalten worden sei. Die wenigen neuen gesetzgeberischen Gestaltungsräume würden kaum genutzt. Das Frühwarn- und Kontrollsystem des Lissabon-Vertrags zur Wahrung des Subsidiaritätsprinzips der EU, das auch den Landtagen neue Interventionsmöglichkeiten bietet, sei weder effektiv noch erfolgversprechend. Seine Einschätzung dürfte leider zutreffen.

Die Kritik an der in der Thüringer Verfassung niedergelegten, automatischen Anpassung der Abgeordnetenentschädigung (Indexierung) ist leiser geworden, nachdem der Thüringer Verfassungsgerichtshof sie als verfassungsgemäß bestätigt hatte. Linck hat an anderer Stelle geäußert, nicht die Indexierung selbst sei das Problem, sondern dass die Entschädigung vor Beginn der Indexierung deutlich zu hoch angesetzt worden sei. ${ }^{2}$

Die Thüringer Praxis ausufernder Zulagen für parlamentarische Funktionsträger ist vom Bundesverfassungsgericht gestoppt worden. Linck kritisiert, das Gericht habe die Bedeutung und Aufgaben etwa der Parlamentarischen Geschäftsführer nicht ausreichend gewürdigt. Mittlerweile hat das Landesverfassungsgericht Schleswig-Holstein Zulagen für Parlamentarische Geschäftsführer der Fraktionen abweichend von der Linie des Bundesverfassungsgerichts für vereinbar mit der Landesverfassung erklärt. ${ }^{3}$

Die Thüringer Verfassung regelt in Art. 67 die Pflicht der Landesregierung, parlamentarische Anfragen zu beantworten und Auskunftsverlangen nachzukommen, sowie die Grenzen der Auskunftspflicht. Linck weist überzeugend nach, dass die Thüringer Verfassung die Grenzen der Auskunftspflicht insofern zu weit zieht, als die Landesregierung die Beantwortung auch dann verweigern kann, wenn der Landtag Vorkehrungen zum Schutz von Staatsgeheimnissen oder des Datenschutzes trifft. Das in Art. 28 Abs. 1 Satz 1 GG verankerte parlamentarisch-demokratische Prinzip verbiete es, bestimmte Materien von der parlamentarischen Kontrolle auszuschließen.

Linck weist darauf hin, dass das Volk, das die Staatsgewalt auf Landesebene durch Wahlen und Abstimmungen ausübt, aus Deutschen besteht und Ausländer nicht darunter fallen. Für die Kommunen sieht Art. 95 vor, dass das Volk eine gewählte Vertretung hat. Klaus-Dieter von der Weiden geht richtigerweise davon aus, dass der Volksbegriff dieser Bestimmung identisch ist mit dem des Art. 45 auf Landesebene. Das von der EU vorgegebene

2 Vgl. Joachim Linck, Wie ein Landtag laufen lernte. Erinnerungen eines westdeutschen Aufbauhelfers in Thüringen, Köln 2010.

3 Urteil vom 30. September 2013, LVerfG 13/12. Vgl. dazu den Beitrag von Siegfried Jutzi in diesem Heft der ZParl. 
und in Art. 28 Abs. 1 Satz 3 GG verankerte kommunale Wahlrecht für EU-Angehörige hat die Thüringer Verfassung nicht inkorporiert, im Gegensatz zu anderen Landesverfassungen. ${ }^{4}$ Dies ist ein Versäumnis, das von der Weiden durch eine grundgesetzkonforme Auslegung lösen will. Nach den geltenden Vorgaben des Grundgesetzes kann ein kommunales Wahlrecht für alle ausländischen Einwohner durch bloße Änderung von Landes(verfassungs)recht nicht eingeführt werden - hier ist von der Weiden zuzustimmen. ${ }^{5}$

Insgesamt ist den Herausgebern und Verfassern ein ausgezeichnetes Werk gelungen, das der Anwendung und dem Ansehen der Thüringer Verfassung in Praxis und Wissenschaft zugute kommt. Die treibende Kraft hinter der Neuauflage war Joachim Linck. Sie ist eine bleibende Erinnerung an sein Wirken.

Florian Edinger

4 Zum Beispiel Art. 50 Abs. 1 Satz 2 LV Rheinland-Pfalz.

5 Vgl. die Berichte und Diskussionen auf der 71. Tagung der Staatsrechtslehrer, in: VVDStRL, 71. Jg. (2012); siehe bereits Katarina Barley, Das Kommunalwahlrecht für Ausländer nach der Neuordnung des Art. 28 Abs. 1 S. 3 GG, Berlin 1999.

Genealogie der Staatsrechtslehre: erhellende Perspektiven auf Wandel und Selbstverständnis des Faches

Schulze-Fielitz, Helmuth: Staatsrechtslehre als Mikrokosmos. Bausteine zu einer Soziologie und Theorie der Wissenschaft des Öffentlichen Rechts, Verlag Mohr Siebeck, Tübingen 2012, 504 Seiten, XX Ausklapptafeln, € 49,--

Farbige Ausklapptafeln sind gewöhnlich in naturwissenschaftlichen oder kunsthistorischen Werken zu finden, in staatsrechtlichen Abhandlungen eher selten. Der diesem Werk beigefügte „Anhang: Ein Jahrhundert deutsche Staatsrechtslehrer“ macht sogleich deutlich, dass Schulze-Fielitz etwas Ungewöhnliches und Einmaliges vorlegt. Bei den mehrfach gefalteten 20 Blättern des Anhangs handelt es sich um eine Art wissenschaftlicher Ahnentafel sämtlicher Mitglieder der Vereinigung der deutschen Staatsrechtslehrer (VDStRL), einschließlich einiger weniger Fachvertreter, die dort nicht Mitglied gewesen sind. Die „Verwandtschaftsverhältnisse“ dieser Personen bestimmen sich nach ihren Lehrer-Schüler-Beziehungen in Habilitations- und Dissertationsverfahren. Ihre Namen nebst Geburts- und Sterbedaten sind, ähnlich wie in Familienstammbäumen, durch sich verzweigende Linien miteinander verbunden, an denen abgelesen werden kann, wer von wem in dieser Disziplin gleichsam abstammt. Der alte Ausdruck „Doktorvater“ lebt mit dieser Graphik wieder auf: Wer hat welche und wieviele Söhne - und in neuerer Zeit auch Töchter - fachlich gezeugt, wie haben diese sich entwickelt, und haben sie ihrerseits Nachkommen hervorgebracht und die wissenschaftliche Familie weitergeführt?

Für die Betroffenen ist das zweifellos interessant und für die Fachkollegen aus den jeweils anderen „Familien“ erst recht. Was lässt sich daraus entnehmen und ableiten, etwa für Status, Rang und Entwicklung des Staatsrechts, für seine soziologischen und (fach)politischen Bedingungen und Wirkungsformen? Schulze-Fielitz listet in einem "Ausblick“ 